\title{
Where Does Cumulative Culture Begin? A Plea for a Sociologically Informed Perspective
}

\author{
Miriam Noël Haidle ${ }^{1,2}$ (1) $\cdot$ Oliver Schlaudt ${ }^{1,3}$ (i)
}

Received: 25 November 2019 / Accepted: 4 April 2020 / Published online: 12 May 2020

(c) The Author(s) 2020

\begin{abstract}
Recent field studies have broadened our view on cultural performances in animals. This has consequences for the concept of cumulative culture. Here, we deconstruct the common individualist and differential approaches to culture. Individualistic approaches to the study of cultural evolution are shown to be problematic, because culture cannot be reduced to factors on the micro level of individual behavior (methodological individualism, "atomism") but possesses a dynamic that only occurs on the group level and profoundly affects the individuals ("holism"). Naive individuals, as a prerequisite of an atomistic perspective, do not exist. We address the construction of a social approach to (cumulative) culture by introducing an inevitable social embedding of the individual development of social beings. The sociological notion of "habitus" as embodied cultural capital permits us to understand social transmission of behavioral components on a very basic level, resulting in a cumulative effect. Bits of information, movement, handling of material, attitudes, and preferences below distinct functional units are acquired through transfer mechanisms simpler than emulation and imitation such as peering, participation, co-performance, or engagement with a material environment altered by group members. The search for a zero point of cumulative culture becomes as useless as the search for a zero point of culture. Culture is cumulative.
\end{abstract}

Keywords Cultural evolution $\cdot$ Cultural niche $\cdot$ Cumulative culture $\cdot$ Habitus $\cdot$ Ratchet effect $\cdot$ Social learning

\section{Introduction}

A broad consensus exists today that behavioral patterns of humans and many other animal species cannot be explained only in terms of genes and the environment, but that there is also a share of culture involved. "Culture" is understood to mean "all behaviors and knowledge that are acquired and passed on within and between generations through social learning" (Schuppli and van Schaik 2019, pp. 1-2; after Boyd and Richerson 1985). In the last two decades, basic cultural capacities (Whiten et al. 2017) have been identified in a number of nonhuman animal species (Whiten 2019) including chimpanzees (Whiten et al. 1999), orangutans (van

Miriam Noël Haidle

miriam.haidle@uni-tuebingen.de

1 Heidelberg Academy of Sciences and Humanities at Eberhard Karls University, Tübingen and Senckenberg Research Institute, Frankfurt/Main, Germany

2 Institut für Ur- und Frühgeschichte und Archäologie des Mittelalters, Eberhard Karls University, Tübingen, Germany

3 Ruprecht Karls University, Heidelberg, Germany
Schaik et al. 2003; Krützen et al. 2011), gorillas (Robbins et al. 2016), cetaceans (Whitehead and Rendell 2014), and New Caledonian crows (Hunt and Gray 2003; Bluff et al. 2010; St. Clair et al. 2016), to name only the most prominent examples. These animals show group-specific differences in social performances, communication, and feeding behavior including tool use. These performances cannot be attributed to environmental conditions alone and thus uncontestably count as cultural. Recently, Schuppli and van Schaik (2019) have markedly extended the perspective on animal cultures, relying on direct evidence for social transmission. Documenting peering behavior by immature orangutans as a process of social learning, Schuppli and van Schaik identified a broad spectrum of performances not previously recognized as culturally transmitted.

The developmental force of cultural capacities observed in nonhuman animals is limited. Searching for a distinctive feature of human as opposed to other animals' culture, scientists have intensely discussed the cumulative aspect and studied living species (e.g., Caldwell et al. 2016; Davis et al. 2016; Dean et al. 2014; Kempe et al. 2014; Tomasello 1999a, b; Vale et al. 2017). These researchers account 
for cumulative culture as a form of behavior that could not have been invented by an individual alone. Rather, cumulative culture represents the modification of existing forms of behavior that were already passed down through social transmission (Boyd and Richerson 1996). Some researchers emphasize the potential of different ways of social learning (e.g., emulation, imitation, over-imitation, forms of active teaching) with regard to social transmission of information and copying fidelity (Tennie et al. 2009; Lewis and Laland 2012). They consider this as a tenet central to understanding culture as cumulative and explaining the high speed of cultural as compared to biological evolution. Many researchers focus especially on the necessity of social learning in tool behavior (e.g., Pradhan et al. 2012; Tennie et al. 2016; Haidle 2019; Stout et al. 2019).

Cumulative culture is usually viewed according to the model of the "ratchet" (Tomasello et al. 1993). In this picture, culture is seen as successively "ratcheted up" from one level to the next. Functionally meaningful units of behavior are transmitted from one generation to the next with high copying fidelity. Occasionally they are modified and these modifications add up to new, higher levels of more complex behavior. Evidence for cumulative culture in nonhuman animals is rare and contested (Schofield et al. 2018). While the question of whether cumulative culture is a purely human phenomenon remains unanswered, it is undisputed that along this path humankind was able to achieve its extraordinary development, leading from basic stone tools to modern high technology.

While there is a consensus on the definition and existence of these cultural phenomena, the question of where culture and in particular cumulative culture exactly begins remains controversial. In many cases there is no consensus on whether an observed behavioral pattern should be considered as cultural —or even cumulative cultural—or not and, consequently, how far the realm of culture extends. In an article from 2009, Tennie, Call, and Tomasello claimed that the realm of culture is much smaller than commonly assumed. They suggested that many behavioral patterns, including the use of tools, actually rely on individual (re-)invention. They envision a "zone of latent solutions" characteristic for each species, which is understood as the scope of things that individuals of a species can easily invent on their own. Certain behavioral patterns would be "latently present in the individual and [are] expressed in the context of specific stimuli or when one recognizes the behavior...expressed by others" (Tennie et al. 2017, p. 652). The idea of latent solutions is that a point in history exists where cumulative effects began to dominate. Before this point, technical performances such as the earliest production of stone tools happened at a purely individual level. Technical skills that are due to latent solutions are determined by the individual's cognitive and motor abilities; they are not culturally transmitted and do not allow for cumulative development. According to this scenario, repeated individual reinvention, rather than social transmission of information, should provide an explanation - and even a much simpler one-of groupwide expressions of many behavioral performances (Tennie et al. 2009). In more recent works, Tennie et al. (2016, 2017) extended this view to early hominins and raised the question of whether individual learning and reinventing in a zone of individual latent solutions might not be a much simpler explanation for the early stone tool industries of the Oldowan (2.6-1.7 Ma before present). According to this view, animal and early hominin performances could be excluded from cumulative cultural capacities and thought of as purely individual achievements. The concept has since been applied in experiments with chimpanzees and modern humans (Reindl et al. 2016; Bandini and Tennie 2017,2019$)$ to explain spontaneously invented solutions to (new) problems.

But does this individualist approach to culture- the focus on discrete and complex behavior and the emphasis on highfidelity forms of social learning - really capture the core of "culture" and thus also the phenomenon of "cumulative culture"? In this article, we challenge the basic assumptions of cumulative culture to gain a better understanding of culture and its cumulative foundation. In the first, critical part, we lay bare the problems, which come along with the basic assumptions of an individualist approach to (human) culture. We show that the individualist approach underestimates the scope of cultural behavior and comes with an individualist bias due to its reliance on the experimental approach. Finally, and most importantly, we demonstrate that the naive individual presupposed by the approach of individual latent solutions does not, and even cannot, exist. From the very beginning, and even before birth, individuals learn by interacting with their environment.

In the second part, we offer a holistic approach with an embedded and developmental perspective. We introduce the notion of "habitus," stemming from Bourdieusian sociology, as a basic cultural and cumulative layer. This requires two aspects. First, we offer an inclusive perspective on the social sphere as an important part of the environment with which the individual interacts and within which the individual develops. The social environment, or cultural niche, can be understood as a supply of social latent solutions. The notion of habitus provides a good framework for a social interpretation of latent solutions. Second, we identify mechanisms of social learning on a non-mentalistic level below the transmission of discrete and complex performances, in particular emulation, imitation, and teaching.

In sum, we reject the individualist reading of latent solutions in basic culture and spell out a generally social reading, which helps us understand that (a) culture is cumulative 
from the beginning and that (b) there is no human nature apart from culture. Putting this discussion in the wider context of the conflict between individualist and holistic approaches to human culture allows us to understand that sociological concepts can also be integrated in theories of cultural evolution and provide a valuable resource.

\section{Deconstructing the Individual and Differential Approaches to Culture}

\section{Individual Performances and Group-Level Phenomena}

Culture and social transmission are phenomena and mechanisms which by definition work on the level of groups and not individuals. These phenomena appear only in groups and can be crucial for the dynamics of these groups. Similar to all disciplines concerned with the realm of human culture, such as sociology, economics, and political sciences, cultural evolutionary studies also encounter a fundamental conflict about how, and in what terms, to account for phenomena at the group level. Can human culture be reduced to factors on the micro level of individual behavior (methodological individualism, "atomism")? Or does a dynamic exist which only occurs at the group level, profoundly affecting the individuals, and thus unable to be reduced to the micro level ("holism")? This conflict has accompanied all of the social sciences since their inception in the 16th and 17th centuries (Freudenthal 1986). For example, modern economics is characterized by a strong individualist consensus, whereas sociology often privileges holistic accounts. The former tends to understand groups as the result of the aggregation of individuals, while the latter considers individuals as shaped by the group. The role model of physics, which is atomistic and mechanistic, exerts a certain influence in favor of individualism. Individualistic psychology looks more familiar to scientists then does its holistic counterpart sociology.

The different views on the beginnings of human culture, cumulative culture versus individual reinventions due to latent solutions, fit neatly into this picture. The appearance of the approach of latent solutions shows that the fundamental question about the right terms to account for culture, individuals or groups, is still far from settled. The strong individualist stance of this latter strand is clearly expressed in the "island test." The island test was introduced by Michael Tomasello (1999a) as a contrastive example to stress the importance of sociogenesis-i.e., the irreducible cultural dimension of development in addition to genes (phylogensis) and individual capacities (ontogenesis). Tomasello intended to emphasize that (cumulative) culture critically depends on cultural transmission and cannot be achieved from scratch by naive individuals on a desert island. "A child raised on a desert island without human companions would not come out as Rousseau envisioned, a 'natural' human being free of the constraints of society, but rather... something of a monster, something other than a fully human intentional and moral agent" (Tomasello 1999a, p. 215).

Tennie et al. $(2016,2017)$ adopt the island test but with a different, even contrary intention. Contrary to Tomasello, who concentrates on more complex skills such as communication through language or other symbolic media, Tennie et al. apply the island test to simple tool production and assume the opposite outcome. They ask the reader to imagine a "naive" individual actually passing the island test: she takes up one stone and hits it on a second one in order to produce an Oldowan-like chopper. How would we account for this outcome of the test? Cumulative culture, relying on some mechanism of social transmission, seems like an excessively and unnecessarily complicated explanation. Was there a latent behavioral pattern that was activated by a kind of impulse? This answer suggests itself as the simplest one and is considered to suit groups of individuals lacking robust mechanisms of social learning equally well.

\section{Experiment and Field Studies}

A first point worthy of elaboration is that the two approaches to culture, individualist and holist, do not necessarily represent an unconditioned choice. Rather, they are tied up with two different paths of investigation: (A) experimentally testing individuals for their capability to solve introduced problems, and (B) the observation in situ of individual and group differences in tool use, controlling the samples for differences in the environments.

Path A represents a simplified situation. It deals with isolated individuals and takes place in the laboratory or in captivity. This setting has the advantage of allowing control over the parameters of the general set-up. It yields a definite answer, yes or no, to the question whether a determinate individual, under determinate circumstances, is able to perform a certain task. The laboratory setting, however, also comes with an important problem. It is not possible to decide whether an individual's achievement is due to innate or acquired skills, a point to which we will return later.

Path B on the contrary tries to capture the individual in situ, i.e., in the field. This allows for studying phenomena in a natural environment and inevitably involves the group level, for conspecifics in general are part of the environment. This approach is also associated with characteristic problems. As often noted, it is extremely difficult to specify criteria for the identification of socially transmitted behavior and thus make a case for culture in animal populations (Galef 1976; Schuppli and van Schaik 2019). 


\section{Culture: Substantial or Differential?}

As a consequence of this difficulty, field studies often rely on what is sometimes called the ethnographic method or the method of exclusion (Whiten et al. 1999; van Schaik et al. 2009). Different populations of a species are compared and controlled for genetic and environmental differences. Variations in behavior count as unambiguously cultural if, and only if, they cannot be accounted for in terms of genes or the environment (Kummer 1971; McGrew and Tutin 1978, p. 241). The classical example is fishing for termites among chimpanzees. The employed techniques clearly vary with the environment, as Tomasello observes (1999b, p. 520): "...the chimpanzees in the western parts of Africa are able to destroy termite mounds with large sticks because the mounds are soft from much rain, whereas in the east they cannot use this strategy because the mounds are too hard." If, however, the environments of two populations do not significantly differ from one another, observed differences in behavior must result from cultural effects within the group. As Schuppli and van Schaik explain (2019), the empirical operationalization of culture as a difference between populations that cannot be explained in genetic or environmental terms subsequently reached the status of a definition, which, for practical purposes, pushed aside the substantial definition of culture as socially transmitted behavior. Consequently Path A and B research temporarily cease to treat the same phenomenon. Path A takes culture as a substantial aspect, yet localizes it in behavioral patterns of individuals. Path B focuses on differences between the behavioral patterns of groups.

The limitation of differences between populations is familiar to behavioral geneticists, who sort genetic from environmental influences on behavior (where the "environment" is everything except the genes). In the case of behavioral genetics, there are important theoretical reasons for constraining its scope to differences between populations. The problem with genes and environment is that these factors do not act independently. To imagine an extreme case, genes without environment would result in no phenotypic expression. As a consequence, and to use a metaphor forged by the primatologist Hans Kummer (1971), asking about the respective contribution of genes and environment to a phenotype is like trying to decide the roles of a drum and a drummer to a drumming sound we hear. This question can be translated into a meaningful one, however, by looking at differences, as Kummer went on to explain:

A person speaks French not only because he grew up among Frenchman, but also because he inherited a genetic basis for language. The trait is neither "acquired" nor "innate" but both. But speaking French rather than Italian can be caused by the environment alone; the difference is purely acquired. Or, in an analogy: It takes a drum and a drummer to produce a sound. Nobody would try to differentiate between sounds produced by the drummer and sounds produced by the drum. But we can very well discuss whether two recorded performances sound different because of a new drummer or a new instrument. (Kummer 1971, p. 12)

If we are interested in explaining differences, it makes sense to ask for the respective contributions of the causal factors. This holds true at least to the degree that the causes can be conceptually separated, as noted by the philosopher Evelyn Fox Keller, i.e., in the picture of the drum, insofar as "the drummer's performance does not depend on the drum being used" (Fox Keller 2010, p. 35). Genetics thus turned from traits to trait differences. Genes ceased to be "trade makers" and instead became "difference makers." At the same time, the focus shifted from the level of individuals to the level of populations. Due to this twofold move, contemporary population genetics, contrary to classical genetics, does not aim to explain traits of individuals, but rather, differences in traits between groups.

At first sight, the problem of the origin of culture seems to be only a special case of the more general problem of nature versus nurture and the respective contributions of genes and the environment (natural or social) to behavior. Indeed, isn't the social environment simply part of the environment, and doesn't it correspond to the original meaning of the term "nurture"? Well, surely it does, but on closer inspection we see that both debates, nature versus nurture and individual versus social, do not share exactly the same structure. Why should techniques of fishing for termites, though differences in them can be accounted for in terms of environmental conditions, not be a cultural trait? Boesch and Tomasello (1998, p. 593) assume that the existence of environmental differences, as in the case of termite fishing, makes individual learning as an explanation more likely. Is this plausible? Techniques of fishing for termites can be cultural and, at the same time, also shaped by the environment.

Van Schaik et al. (2009) and Schuppli and van Schaik (2019) make this fundamental point: social learning need not result in differences between groups. Furthermore, differences between groups, even if they can be accounted for in terms of genetic or environmental differences, do not exclude that social learning is involved. Finally, if there is simply no group to compare with, many other cultural phenomena may simply remain unnoticed. Cultural phenomena detected through the method of exclusion thus represent only a "small and highly biased subset of what we naturally claim as part of our culture" (Schuppli and van Schaik 2019, p. 4). 
The comparison with the classical nature versus nurture debate can help establish this point with logical rigor. Despite the similarities, an important difference between both debates remains, which contrary to genetics, suggests that the quest for (animal and human) culture is not confined to differences. The important point is that we do not ask for the respective share of nature and culture in given traits, but rather whether social learning is involved at all. If so, the trait counts as cultural, not individual. Or in order to stay in Kummer's picture, the question is not about what part the drummer plays in making the noise, but about whether a drummer is involved at all or, if instead, the noise merely results from a bar dangling loosely in the wind.

Based on Schuppli and van Schaik's ideas and contrary to population genetics, the investigation of culture can expand beyond differences and engage in a "substantial" notion of culture as socially transmitted behavior. The importance for our case can hardly be overestimated. On the one hand, this puts Path B on a par with Path A regarding the underlying concept of culture. Indeed, what is at issue with both approaches is the substantial notion of culture. On the other hand, this shift in definition of "culture" undermines much of the empirical evidence for latent solutions. Tennie et al. (2009) constantly rely on the method of exclusion and the related differential notion of culture in order to downplay cases of social transmission. They acknowledge culture only where differences cannot be accounted for in terms of the environment. But this is clearly a mistake. That variation in behavioral traits can be explained in terms of the environment does not exclude that social learning is involved in them, as we have clearly seen.

\section{Group-Level or Individuals?}

The shift of perspective on "culture" by Schuppli and van Schaik puts Paths A and B research on a par regarding the underlying notion of culture. However, this shift does not change everything. The observational approach of Path B is still concerned with groups, not individuals, and this will turn out to be critical.

As stated above, the experimental approach of Path A, dealing with isolated individuals in captivity, makes it difficult if not impossible to decide whether a given achievement is due to innate or acquired skills. In laboratory experiments, individual ability and social heritage become indistinguishable, as every output appears as an individual achievement. This represents a potential individualistic bias, which becomes a real problem once the experimental results are projected without qualification on the natural condition outside the laboratory. This is what actually happens in the thought experiment of the island test. The scenario of the island test indeed merges aspects of both paths of research. It deals with individuals as in Path A experiments, but imagines the individuals in their natural environment as in the Path B approach. It projects without qualification the individualist framing of Path A research onto the situation outside the laboratory, and also back in time onto early hominins in prehistory, as studied in Path B research.

This is a risky enterprise and comes with two major problems. First, it takes the population level simply as reducible to the level of individuals without social and developmental dimensions. The cultural approach to tool use and cognition on the contrary explains these phenomena in terms of mechanisms that only emerge on the population level. Second, the individualist approach, to be meaningful, presupposes the notion of naive individuals, as Tennie et al. (2017) stress. In the experimental work linked to the approach of latent solutions (Tennie et al. 2009; Reindl et al. 2016; Bandini and Tennie 2017, 2019), an individual, human or nonhuman, counts as naive when he or she is not familiar with the special task tested in the experiment. However, what about the prior experiences the individual has had during his or her life, and what about the collective experiences on a historical scale, which are stored up in the design of the artifacts used in the experiment? This line of thought invites us to raise the general question of whether one can think of any circumstances at all under which the assumptions of a "naive" individual in an experimental setting seem legitimate.

\section{Naïve Individuals Do Not Exist}

The assumption of "naive" individuals has always provoked sharp criticism. Claude Lévi-Strauss commented as early as 1949 on the problems accompanying such attempts to carve out human nature as opposed to culturally shaped traits. He says ([1949] 1969, p. 4): "The simplest method [to decide whether observed behavior is cultural or natural] would be to isolate a new-born child and to observe its reactions to various stimuli during the first hours or days after birth." However, since many capacities like walking show up only later in life when the underlying physiological mechanisms are fully developed, Lévi-Strauss argued that isolation would have to be extended over several months or even years. He concluded, “...the environment satisfying the strict isolation requirements of the experiment is no less artificial than the cultural environment it purports to replace, in that, for example, during the first years of life, maternal care is a natural condition in the individual's development. The experimenter is locked in a vicious circle" (Lévi-Strauss [1949] 1969, p. 4). "Man himself," Lévi-Strauss summed up, "cannot be expected to exemplify types of precultural behaviour" ([1949] 1969, p. 5).

Should we deduce from this that we cannot decide whether a given trait or behavior stems from individual latent solutions or cultural transmission? Lévi-Strauss' 
objection seems to be even more radical, for he does not simply call into question the empirical foundation of our knowledge of human nature, but suggests rejecting the whole idea of natural traits, given that there is simply no environment for humans that could be qualified as natural. "Culture is the human nature," as Sahlins more recently put it (2008, p. 110). We think that Lévi-Strauss' ideas hint at the correct direction for a critical discussion of latent solutions. Individual latent solutions can be identified only through the behavior of naive individuals, but these, as Lévi-Strauss suggested, do not exist in social beings and, moreover, cannot exist. The naive individual is not only counterfactual, but inconceivable. This, we would like to add, applies to humans as well as orangutans and all other species raised in a social context.

Shortly after Lévi-Strauss, the animal psychologist Daniel S. Lehrman expressed a similar critique of Konrad Lorenz' notion of instinctive behavior, thus opening up a new chapter in the nature versus nurture debate (Lehrman 1953). Instinctive behavior has much in common with individual latent solutions, in particular that they are (partly) hereditarily determined and appear in organisms raised in isolation from others. Several examples showed that alleged innate behavior often turns out to be acquired as a result of the animal's interaction with its environment. The pecking behavior of chicks turned out to originate from tactual stimulation of the embryo in the egg. The embryo's heartbeat stimulates tactually the head during the first days of the embryonic development, and shortly after, the head starts to bend actively in response to tactile stimulation. Similarly, nest building behavior of pregnant rats, which occurs in individuals raised in isolation and thus seems to be a candidate for innate behavior, turns out to depend on their previous experience carrying objects. Rats that had been prevented from manipulating solid objects (e.g., by providing them only with powdered food) proved unable to build nests out of strips of paper or other material. These examples show that alleged innate behavior may in fact result from interaction with the environment, and that this interaction can occur extremely early in the development of the organism, even before birth.

Consequently, Lehrman formulated a general critique of isolation experiments. Isolation experiments can isolate animals only from certain environmental factors, but not from all of them, because organisms cannot live, let alone grow and develop, in a vacuum. There is no full isolation, and hence it is vain to prove by means of isolation the innateness of certain behavioral patterns (Lehrman 1953, p. 343). Since organisms need an environment and from the very beginning develop in close interaction with it, the whole dichotomy of heredity and environment, or nature and culture, proves to be artificial, as Lehrman noted (1953, p. 345). This brings us back to the caveat formulated by Evelyn Fox Keller for behavioral genetics. Trying to assess the share of the drummer and the drum with regard to a difference in sound only makes sense if we can separate the drummer and the drum, that is, if the drummer's performance does not depend on the drum used. It is this assumption that turns out to be problematic when we realize that there cannot be a "naive" drummer. There can only be drummers who have already been trained on drums that actually constitute their environment and forged their drumming skills. A strict separation between the drummer and the drums becomes meaningless, and so does the idea of a pre-cultural individual.

\section{Constructing the Social Approach to (Cumulative) Culture}

\section{A Socially Embedded Perspective}

We have already seen in Lehrman's examples that a fundamental level of learning exists, through which early interaction with the environment results in competences. This was traditionally interpreted as innate. Whereas Lehrman's examples were confined to purely physical aspects of the environment, the recent literature provides impressive evidence for similar cases of alleged innate behavioral patterns that turn out to be induced by aspects of the social environment. The migration patterns of bighorn sheep and moose, for example, evolve by social learning as a primary mechanism (Jesmer et al. 2018): neither emulation nor imitation is involved in the process, but rather the embodied experience of unacquainted individuals who simply accompany adept ones. Homing pigeons have demonstrated the ability to accumulate progressive modifications across multiple generations, not through individual cognitive complexity but by learning to follow the group and combining their collective intelligence (Sasaki and Biro 2017). Observational social learning in combination with socially induced practice over a period of several years is a critical component of the acquisition of learned subsistence skills in orangutans (Schuppli et al. 2016; Schuppli and van Schaik 2019).

In all of these examples, young individuals grow up in social groups that constitute part of their learning environment. From birth on, they are introduced to group habits by exposure to the same (social and material) environment as the other group members, invited to engage with similar things, problems, and solutions. They constantly interact with and learn from their environment through their actions, perceptions, and cognitive abilities (Streri et al. 2013). Infant chimpanzees who cling to their mother 24 hours a day move around with her and are exposed to stimulation mediated by the mother's behavior. Infants are imbued with fundamental action patterns-rhythms of movement and rest, moods such as stress, anxiety, calm, and joy, patterns of 
social interaction and of interaction with the material environment—even before they start to perceive their mothers' actions as controlled manipulations of objects and thus even before they could start to imitate them. They are much more likely to eat what their mothers eat, to move when and where the group members move, to communicate in ways and at instances when the others do, to handle those objects the group members handle, and to explore the segment of the environment that the group occupies (Whiten and van de Waal 2018). They share experience of knowledge about locations, options, timing, adequacy, and additional needs, of emotions and rhythm. The closer and more stable social bonds are within a group, the more experiences can be shared, and the more habits are consolidated.

As in Lehrman's examples, the process of social transmission of behavioral patterns already begins in the uterus. Embryologists have long recognized that (1) the (human or nonhuman) fetus is not deprived of sensory stimulation (Colombelli-Négrel et al. 2014; Hepper 2015) and (2) the motor activity of fetuses is not simply an epiphenomenon of the developing nervous system, but rather exhibits organized patterns associated with presumptive sensory events (Smotherman and Robinson 1990). Individuals are not "age zero" at birth, as Hepper (2015, p. 38) stresses, but have passed through a period of embryonic learning in the intrauterine environment. We would only add that the uterus has to be considered as a social environment. Indeed, among the prenatal experiences and stimuli are those which in particular are connected to maternal behavioral patterns. The influence of the mother's behavioral patterns during pregnancy extends far beyond purely biological and physiological effects and conditions the fetus' behavior. Examples are the shaping of food preferences by maternal diet during pregnancy (Anzman-Frasca et al. 2018), temperament by prenatal exposure to maternal stress (Davis et al. 2007), and postnatal auditory preferences by maternal sound and movement patterns (Moon 2017). These are impressive examples of a prenatal transmission of information on a sub-imitation or sub-emulation level. Even at birth, infants are already far from being the naive individual presupposed in the individualist reading of latent solutions. They carry a matrix of possible actions, which, though not determining their behavior, suggest certain patterns and preclude others.

Moreover, humans are characterized by the fact that, due probably to the obstetrical dilemma (e.g., Rosenberg and Trevathan 1995), most brain growth occurs after birth while the child interacts with the natural, cultural, and social environment. They are "secondarily altricial," to use a term coined by Portmann (Portmann 1941; cf. Gould 1976; Hublin 2003). This means that not only do we have to consider the uterus as a social environment, as explained above, but, vice versa, we see that the postnatal social environment works as a functional equivalent of the uterus: the community is a kind of social uterus. Human offspring comes to maturity in a social and culturally saturated environment, which the immature child incorporates. This peculiarity of humans thus gives a new and unknown importance to the third, sociogenetic developmental dimension of cultural capacities (Haidle et al. 2015).

These examples have two important consequences. First, they clearly show that the social sphere is part of each individual's environment, understood as a resource space for learning situations. This holds even before birth, for the intrauterine environment also has a social dimension, as we have seen. As a second consequence, we see that group-specific practice patterns are transmitted at a sub-action level. Since the practice patterns are not recognized as actions, i.e., as manipulations of objects by actors, both emulation and imitation fail as mechanisms of transmission. Here a social interpretation of latent solutions on a group level comes into play. Couldn't we think of group-specific practice patterns as the cultural nutrient medium for latent solutions of problems, which are characteristic of the group's specific environment and which can be activated by individuals in their behavior? To see what a social interpretation of a zone of latent solutions can mean, we have to broaden our view on cultural performances as well as cultural transmission.

\section{Habitus: Socially Embodied Cultural Capital}

Since we already stressed the importance of the sociogenetic dimension in the development of cultural capacities, it may come as no surprise that a concept stemming from sociology may prove helpful and integrate with biological theories in a natural way. Pierre Bourdieu's term "habitus" (Bourdieu 1977) or "embodied cultural and social capital" (Bourdieu 1986) is a good candidate. ${ }^{1}$ This notion has occasionally attracted attention in cognitive archaeology (de Beaune 2016, p. 138; Malafouris 2013, p. 141) and in cultural niche construction theory, understanding habitus as a part of the social and cultural niche (Kendal 2011, p. 245; Fuentes 2016, p. S18). To our knowledge, however, no attempts have been made to systematically exploit it. The French sociologist originally forged these concepts with a

\footnotetext{
1 Strictly speaking the notion of habitus represents only one corner of a triangle of three concepts that belong together methodologically and permit Bourdieu "the triple historicization of the agent (habitus), the world (social space and fields) and of the categories and methods of the social analyst (reflexivity)" (Wacquant 2016, pp. 64-65). For the purpose of this article it seems legitimate to focus on habitus alone, setting aside the two other concepts, and thus to reduce habitus to embodied cultural capital. Incidentally, "embodied" is the term that Bourdieu himself used. This happened long before the approach of embodiment in the cognitive sciences arose. In our opinion, these are not false friends, but compatible approaches, as we maintain in the text.
} 
view to accounting for class-specific behavioral differences between individuals acting in the same social environment. The classical example is children's performance in public schools. The possession of embodied cultural capital permits us to understand the above average results of upper-class children in school, i.e., in a context where differences in economic capital cannot be of direct influence. Bourdieu (1977, pp. 82-83; italics in original) defined habitus as

a system of durable and transposable dispositions which, integrating all past experiences, functions at every moment as a matrix of perceptions, appreciations, and actions, and makes possible the achievement of infinitely diversified tasks, thanks to analogical transfers of schemes permitting the solution of similarly shaped problems....

As Loïc Wacquant further explains (2016, p. 66), habitus "designates a practical competency, acquired in and for action, that operates beneath the level of consciousness and is continually honed in the very movement of its deployment." The habitus thus does not show the structure of an action, but is located on the sub-action level of dispositions and competency, which determines the individual's overall relation to his or her environment. It specifies which resources of the environment can or cannot be put into action, and thus defines the individual's space of possible actions. It roughly corresponds to the stance individuals takes vis-à-vis their environment: are they bold or shy, riskseeking or risk-averse; which resources and opportunities are they able to identify and put into action for their own benefit? Perhaps, the notion of habitus is not dissimilar to what Darwin described as "attitude," to quote a concept stemming from the history of biological theory (Darwin 1872; cf. Barsalou 2003, p. 63). Although Bourdieu forged the notion of habitus for the analysis of modern, functionally differentiated and socially stratified societies and with a view to explaining the persistence and reproduction of social inequalities, we see no obstacle in applying this concept even to less differentiated small groups in deep history or socially raised animals. In this case, embodied cultural capital does not account for the reproduction of in-group inequalities, but would describe a group-specific, collectively shared, and socially transmitted stance towards the environment.

The social transmission and the development of habitus are crucial points to understand the effectiveness of the habitus in cultural evolution and its relevance for cumulative culture. Bourdieu, in his study of French high school students, referred to the mechanism of osmosis as a model for an unconscious and effortless process during which behavioral patterns seep into the organism: "Thus, lycée pupils from the Paris bourgeoisie are able to manifest an extensive culture, acquired without intention or effort, as if by osmosis, at the very moment when they are denying that they experience the slightest parental pressure" (Bourdieu and Passeron 1979, p. 20). The unconscious character of the transmission of the habitus plays an important role in the explanation of social inequalities in contemporary societies. In Bourdieu's example, the transmission of cultural capital works smoothly exactly because parents do not understand it as an investment, but think of culture as a value in itself. School teachers reward the performance of upper-class children exactly because they understand it as a matter of individual giftedness, not social heritage (i.e., teachers behave like ethologists with the individualist bias of the Path A view!). Of interest in this context is that, as mentioned above, habitus was equated by several authors with social niches, and that the niche was understood by them first and foremost as a learning environment.

As niche construction, a phenomenon is described by which

organisms, through their metabolism, their activities, and their choices, define, partly create, and partly destroy their own niches... [N]iche construction regularly modifies both biotic and abiotic sources of natural selection in environments and, in so doing, generates a form of feedback in evolution.... (Odling-Smee et al. 1996, p. 642; cf. Levins and Lewontin 1985)

Cultural niche construction puts special emphasis on the role of culturally acquired traits in the transformation of the selective environment (Laland et al. 2000; Laland and O'Brien 2011; Odling-Smee and Laland 2011; Rendell et al. 2011). For our purpose, it is central that the cultural niche not only feeds back on gene selection, but also opens up direct channels of cultural inheritance via transmission of information through the physical, social, and cultural environment. "Human ecological inheritance is exceptionally potent because it includes the social transmission and inheritance of cultural knowledge, and material culture" (Kendal et al. 2011, p. 785). Diverse authors stressed in this sense that the (pre- and postnatal) niche works as a learning environment and specifies behavioral adaptions (West and King 1987; Ambrose 2010; Kendal 2011). Learning can be understood here at a sub-imitation level. West and King (1987, p. 558) remind us that "care-giving and culture-giving go hand-in-hand," while Kendal (2011, p. 242) hints to "situated learning" understood as "learning in situ through participation."

Regarding the question of cumulative culture with its aspect of sequential cultural changes, it is of special importance to take the sociogenetic perspective on the development of the niche itself and performances within. The model of the Evolution and Expansion of Cultural Capacities (EECC) (Haidle et al. 2015) combines three developmental dimensions of organismic performances interacting with each other and the specific environment, or rather, cultural 
niche. The specific environment is a selective environment constantly modified by organismic performances. In a similar spirit, in the early 20th century the Soviet developmental psychologists Vygotsky and Luria put forward the thesis that the behavior of humans has to be understood as the product of three principal lines of development: "the evolutionary, the historical, and the ontogenetic" (Luria and Vygotsky 1992, p. xi). In the EECC model, the evolutionary-biological dimension refers to genetically inherited basics of performances such as general anatomic structure and metabolic processes, which change through mutation and selection. In constant interaction with their specific environment and resulting individual experiences, an organism individually learns and develops epigenetic responses. Thus, the performances of an organism unfold in the ontogenetic-individual dimension, with invention and habituation as well as preference and avoidance as drivers. In social organisms, however, conspecifics and their performances have an additional share in the specific environment, which constitutes the developmental space. The individuals not only learn from their own experiences, but also socially from the experiences of group members, manifest in their behavior and products. This historical-social dimension develops through processes of shift in the common group behavior resulting in a constant conflict and alternation of innovation and tradition. The organismic performances represent interactions with the specific environment comprising conspecifics and other biotic and abiotic agents as well as objects in specific relationships and certain time-depths. It is a cultural niche including the performances of group members and their products as factors as well as effects of the historical-social dimension of development (Haidle et al. 2015). The habitus with performances simpler than functional behavioral units is an important part of this learning environment.

\section{Example "Stone Tool Production": Habitus in Early Hominins}

Let us discuss the specific example of early stone tool production in hominins to illustrate the advantages of our analytical framework. An individualistic setting such as the island test used by Tennie et al. $(2016,2017)$ takes performance as a discrete functional unit, that is, as an appropriate solution to a well-defined problem. In this picture, naive individuals take up two stones, pound them against each other, and produce flakes with sharp cutting edges, thus inventing repeatedly the process of manufacturing flake tools. The perception of the problem and the respective sequence of actions towards a solution are taken as a discrete package, distinct from other problem-solution combinations. Examining the transfer of a problem-solution setting focuses generally on the process of learning the entity, as increasing fidelity in copying shows an increasing degree of sophistication.

Applying the concept of habitus to the case of early hominin stone knapping, in contrast, takes a holistic view. As part of the cultural niche to which the offspring is exposed, the habitus does not consist of discrete functional units. Rather, it is composed of many small bits of skill and knowledge, acquired by individuals during a lifetime through material and social engagement in a socially formed learning environment. From an archaeological perspective (focusing mainly on tool behavior), habitus includes general problem perceptions and implicit knowledge about specific resources, their qualities and application, timing and pattern of acquisition, pathways, locations, and tools as well as practices of their manufacture, use, maintenance, and discard. This makes sense when applied to stone knapping. Stone knapping includes many more aspects than just pounding stones against each other, as capuchin monkeys do (Proffitt et al. 2016; see also Lombard et al. 2019). Not every rock is capable of producing flakes with sharp edges, not every nodule is appropriate, and suitable ones are not available everywhere. The same applies to hammerstones of adequate size and weight. The practice of deliberate manufacture of flakes needs some knowledge about combining raw material and tools, plus lengthy learning of knapping skills, which requires the handling of items different than those required for mere pounding or nut cracking. Additionally, the application of the products in a cutting task requires a general awareness of the possibility of the task, some familiarity with the qualities of the target object, its location, and acquisition, as well as know-how and practice in tool use. The idea of a spontaneous invention of all these different know-that/ how/where/whens and respective skills by one individual is not only unrealistic, it also emanates from the false notion of naive individuals. Individual learning becomes much easier when individuals can build on group habits such as tool use. This may include pounding behavior with stones, knowledge about raw material and its location, and preference for meat as a food resource, which becomes more accessible through cutting. Communal hunting and eviction of enemies or competitors could have facilitated the capture and defense of carcasses to which the cutting tools were applied in order to dismember them. All of these things could have been part of the habitus allowing for adjacent social latent solutions. If we shift our focus on cultural traits from large functional units to more subtle elements of skill and knowledge, the perspective on cultural transmittance expands towards a fundamental level simpler than emulation and imitation, and the zone of latent solutions changes.

The introduction of each of these aspects to the habitus of the group altered the zone of social latent solutions. Once the use of cutting flake tools had been included in the standard behavioral repertoire of some group members, 
unacquainted individuals shared experience, thus fostering the adoption of the performance and modifying again the set of latent solutions, this time, for example, the possibility of cutting things other than meat. The habitus is a socially embodied cultural capital that has opened new potential performances, that is, latent solutions (and latent problems). The habitus, a group-specific matrix of perception, appreciation, and action, forms the basis of group-specific latent problem perceptions and solutions, which is close to the already existing performances of the group (Haidle 2019). Conceived of as group specific and social, the concept of latent solutions no longer contrasts with cultural transmission and cumulative culture, but represents a vital part of it.

\section{A Developmental Perspective}

As the example of stone tool production shows, the approach of habitus first and foremost implies a shift in the analytical units of behavior, from discrete functional units of appropriate solutions for well-defined problems to smaller elements of skill and knowledge. This shift has two consequences. First, it makes the model of osmosis-like absorption plausible. The components of behavior we are looking at are not functional units, which can be acquired via emulation or imitation. They are generally, as in Bourdieu's example of the pupils, not taught. They are rather a cultural repertoire of small bits of practice and information, which the individual adopts through mere habituation. Accompanying their close social partners, they are introduced to selections of preferable qualities of situational, material, physical, mental, emotional, and behavioral conditions and opportunities, or limitations to change these. If unacquainted individuals get access to tools of proficient ones, they can experience and learn individually by relying on activities and experience of the "experts," as manifested in the qualities of the artefacts. If some raw material sources are favored and others ignored, the less informed individual incorporates this preference. Independent of direct individual threat, an unexperienced subject learns from the reactions of group members whether encounters with other organisms in specific situations are potentially dangerous, neutral, or even fruitful. The less knowledgeable and skilled individuals learn all of these things, and much more, in a social context, neither as discrete functional units with a clear focus on a specific problem (as in emulative learning), nor as mental templates of the solution of a problem (as in imitative learning). Rather, they absorb the different elements of the habitus of their social group by making their own experiences via participation in performances of others and in a socially preselected environment. They incorporate it through social engagement and engagement with culturally loaded material (cf. Malafouris 2013). Thus, they learn individually and simultaneously embody the socially provided defaults, the habitus, as a basal layer for any activity, sense-making, or attitude to face new challenges. The unacquainted individuals develop their performances through the interaction of ontogeneticindividual and historical-social dimensions within the culturally formed specific environment or niche. The habitus is part of this specific environment, which in fact constitutes a resource space or zone of social latent solutions.

Once an individual develops a new variant of a performance-another movement, another timeframe, another emotional loading, another raw material, another application-and includes it in her/his standard repertoire, other group members share in it at least partially via peering, participation, co-performance, or an altered material environment (for example, see learning mechanisms in New Caledonian crows: Logan et al. 2016, p. 26). Unacquainted individuals develop their own performances within this modified learning environment. Proficient individuals, however, can also adapt to new elements of the habitus. This is not restricted to humans, as exemplified by migrating female chimpanzees that changed their selection of nut-cracking tools according to the preference of a new group (Luncz et al. 2015). Individuals, habits, groups, and habitus all coevolve. Based on their previous experiences in a socially affected environment (cultural niche), individuals develop their performances and habits with a certain group conformity, forming a specific habitus that is reinforced by individual performances or works as a basis for new performances. These can serve as the seed for a modified habitus. Modifications begin on the individual level (invention) and can spread within the group (innovation) under favorable conditions (Leroi-Gourhan 1945; de Beaune 2015). Alterations of the individual (invention) or the group level (innovation) can be very subtle and do not always represent completely novel functional units (Haidle and Bräuer 2011). The change of habits in individuals and their diffusion into the habitus of groups is a process of diffusion, in which new elements and well-known traits compete. Innovations have to be established in a broader context of a variety of often changing interests, supportive factors, and constraints (cf. Rogers 1995). Often it is not the urgency of the problem that is lacking, or the quality of the solution that lets an innovation fail, but rather the habitus that has to change first.

This crucial role of habitus in processes of innovation also implies a criticism of the ratchet effect, a central aspect of the concept of cultural evolution "in which modifications and improvements stay in the population fairly readily (with relatively little loss or backward slippage) until further changes ratchet things up again" (Tennie et al. 2009, p. 2405). The model of the ratchet works on the level of discrete functional units of behavior, which are transmitted with high fidelity and are occasionally modified resulting in improved solutions and an increase of complexity. However, from the perspective of habitus as a basic cultural 
repertoire, the modeling of the course of cumulative cultural development as a ratchet has to be reconsidered. As we argued above, cultural performances can represent discrete functional units from an analytical perspective. However, neither in the individual run nor at the group level do cultural performances represent developmental entities. They consist of a multitude of bits of information, movement, handling of material, attitudes, and preferences acquired over time in various situations with substantial fidelity, yet mostly on a transmission level below emulation, imitation, or teaching. Additionally, every performance entails multiple effects, which can be positive, negative, or neutral. A new performance can thus represent an improvement from some perspective but a deterioration from another, as illustrated by numerous examples in the work of Rogers (1995) on the diffusion of innovations. To overcome this problem in the concept of cumulative cultural evolution, Lombard (2016) suggests a "mountaineering effect" instead of a ratchet effect of increasing fitness or improvement. Following this metaphor, the development is path-dependent or sequential, as novelties depend on previous developments and facilitate or hamper further steps in certain directions. Each step discloses new perspectives, which alter the effects of the performance by reducing some and increasing or shifting others. Habitus is the current standpoint of the individual and its group in the landscape (specific environment, niche), reached by a sequence of a multitude of small choices, fortuities, and subsequent steps, and offering perspectives in different directions for further performances.

\section{Conclusion: Consequences for Cultural-Evolutionary Studies}

Mesoudi and Thornton (2018, p. 2) state that,

the minimum requirements for a population to exhibit CCE [cumulative cultural evolution] are (i) a change in behaviour (or product of behaviour, such as an artefact), typically due to asocial learning, followed by (ii) the transfer via social learning of that novel or modified behaviour to other individuals or groups, where (iii) the learned behaviour causes an improvement in performance, which is a proxy of genetic and/or cultural fitness, with (iv) the previous three steps repeated in a manner that generates sequential improvement over time.

Mesoudi and Thornton's core set of criteria for CCE include the ratchet model through the word "improvement" in point (iii). We instead suggest path-dependency as a criterion, as presented in Lombard's more realistic model of mountaineering. In this case, the concept of habitus and its development, as sketched in this article, fit very well with this definition of cumulative cultural evolution: (i) Changes of individually developed behavior (ii) modify the socially formed material and social learning environment by which the novel behavior is transferred to other individuals. (iii) The learned behavior causes alterations in performance that disclose and create new possibilities and limitations for further development. (iv) These steps are repeated in a manner generating sequential development over time. Individuals of cultural species build their performances on the behavior and its products of group members. They have history and cultural evolution (Schofield et al. 2018, p. 120). As a conclusion, let us spell out the consequences for research in cultural evolution.

\section{Cultural Evolution in Early Hominins}

A first consequence of our approach is that culture in early hominins has to be regarded as cumulative from the very beginning. The search for a zero point of cumulative culture is as useless as the search for a zero point of culture. As we pointed out, a nonsocial individual nature in social beings does not exist. They are cultural, although they may not express the cultural capacities of humans today (Haidle et al. 2015; Löffler 2019). The ontogeny of social beings unfolds in socially mediated settings that form the learning environment. We consider early hominins as living in groups with social bonds more or less comparable to other primates, thus passing through individual ontogenies with learning phases that are inseparable from interactions with the social environments (cf. Whiten and van de Waal 2018). Therefore, we have to assume a specific social habitus. This habitus is a zone of socially induced latent solutions; it provides a set of information, practices, and preferences acquired over time as defaults that form the basic layer for any activity, sense-making, or attitude to face new challenges. Together with the structure and bonds of the social groups and their interactions with their specific environment, the habitus of early hominins and their zones of latent solutions constantly developed. In human evolution, an expansion of mechanisms of transmission of social information unfolded, accompanied through an increase in copying fidelity: from embodied shared experience, stimulus enhancement, and response facilitation with elements of reinvention via emulation, imitation, and over-imitation to various forms of transfer with active engagement of an expert. However, the introduction of new transmission mechanisms does not mirror a clear-cut difference of additive accumulation of traditions. This is opposed to cumulative culture building upon earlier achievements, but allows an increase in developmental flexibility and velocity. 


\section{Cultural Evolution in Modern Animal Species}

The same is true for the cultural evolution in modern animal species such as great apes, cetaceans, and New Caledonian crows, but also herding animals such as large ungulates and flocking birds. Again, we have to accept a certain social habitus and consequently a certain set of socially induced problem perceptions and latent solutions. The longer the learning phase in ontogeny, the closer the social bonds within the groups, and the richer the interactions with the material environment. This creates a more diverse social habitus, a broader zone of (socially induced) latent solutions and, accordingly, the potential for cultural development. In social beings, there is no dichotomy of environmental shaping in contrast to cultural learning, as social interactions are an integral part of their environment and forming it. The search for features discriminating between animal and human culture helps neither in studying the diversity of cultural capacities and performances, nor in understanding the variety of aspects in the processes of cultural development. Exploring the range of cultural capacities and their cumulative potential will yield a fascinating array of different expressions and mechanisms, as shown by studies on food-washing by Japanese macaques (Schofield et al. 2018), the migration of bighorn sheep and moose (Jesmer et al. 2018), and the development of homing routes in pigeons (Sasaki and Biro 2017). This endeavor to trace the course of development from individual novelties, to innovations, to traditions and the mechanisms behind those, however, needs a long-term perspective of several years up to decades.

\section{Experimental Studies}

Following the remarks above, experiments focusing on individual capacities for invention are not apt to explain behavioral patterns in groups. Additionally, we cannot expect fundamentally naive individuals without any form of socialenvironmentally mediated experience. Even if an individual and his or her social group have never been confronted with a specific task, the test individual is not completely naive in this respect, but relies on former embodied shared experiences and learned performances from other contexts. Consequently, experimental studies cannot falsify the hypothesis of a (cumulative) cultural element in the development of a performance. However, experimental studies can provide insight into different mechanisms of cumulative development and their effective range (cf. Gruber et al. 2019; Logan et al. 2016; Vale et al. 2017; Caldwell et al. 2020).

\section{Shift in Research Questions}

Trying to discriminate purely individual versus culturally mediated performances (be it in modern humans, modern animals, or early hominins) and to separate cultural from cumulative cultural performances has shown to be grounded upon false assumptions. Instead, we should investigate processes and modes of creativity beyond small-scale individual variation and the integration of advanced elements into existing traditions or cultural patterns. Differences in these aspects of cumulative culture between primates and other animal species are likely to yield more insight into processes at the dawn of hominization and the development of modern social species than discriminating studies between modern great apes and modern humans.

It is time to rethink the cumulative aspect as a general trait of culture. Cultural capacities are an inseparable factor in the development of social beings expressed in different grades of historical-social depth and patterning (cf. Haidle et al. 2015). This does not take the study of cultural evolution ad absurdum, but opens a large field of yet unexplored questions.

Acknowledgments Open Access funding provided by Projekt DEAL. The authors are grateful for the financial support by the Academies' Program of the Union of the Academies of Germany and the Deutsche Forschungsgemeinschaft. We appreciate the comments of two anonymous reviewers, which helped to sharpen our arguments.

Author Contributions Both authors contributed to development of ideas, writing, and editing to the same extent.

Open Access This article is licensed under a Creative Commons Attribution 4.0 International License, which permits use, sharing, adaptation, distribution and reproduction in any medium or format, as long as you give appropriate credit to the original author(s) and the source, provide a link to the Creative Commons licence, and indicate if changes were made. The images or other third party material in this article are included in the article's Creative Commons licence, unless indicated otherwise in a credit line to the material. If material is not included in the article's Creative Commons licence and your intended use is not permitted by statutory regulation or exceeds the permitted use, you will need to obtain permission directly from the copyright holder. To view a copy of this licence, visit http://creativecommons.org/licenses/by/4.0/.

\section{References}

Ambrose SH (2010) Coevolution of composite-tool technology, constructive memory, and language. Curr Anthropol 51:S135-S147

Anzman-Frasca S, Ventura AK, Ehrenberg S, Myers KP (2018) Promoting healthy food preferences from the start: a narrative review of food preference learning from the prenatal period through early childhood. Obes Rev 19(4):576-604

Bandini E, Tennie C (2017) Spontaneous reoccurrence of "scooping", a wild tool-use behaviour, in naïve chimpanzees. PeerJ 5:e3814

Bandini E, Tennie C (2019) Individual acquisition of "stick pounding" behavior by naïve chimpanzees. Am J Primatol 2019:e22987

Barsalou LW, Niedenthal PM, Barbey AK, Ruppert JA (2003) Social embodiment. In: Ross BH (ed) The psychology of learning and motivation, vol 43. Academic Press, Amsterdam, pp 43-92

Bluff LA, Kacelnik A, Rutz C (2010) Vocal culture in New Caledonian crows Corvus moneduloides. Biol J Linn Soc 101(4):767-776 
Boesch C, Tomasello M (1998) Chimpanzee and human cultures. Curr Anthropol 39(5):591-614

Bourdieu P (1977) Outline of a theory of practice (Richard Nice, trans.). Cambridge University Press, Cambridge

Bourdieu P (1986) The forms of capital. In: Richardson JG (ed) Handbook of theory and research for the sociology of education. Greenwood, New York, pp 241-258

Bourdieu P, Passeron J-C (1979) The inheritors. French students and their relation to culture (Richard Nice, trans.). University of Chicago Press, Chicago

Boyd R, Richerson PJ (1985) Culture and the evolutionary process. University of Chicago Press, Chicago

Boyd R, Richerson PJ (1996) Why culture is common, but cultural evolution is rare. Proc Brit Acad 88:77-93

Caldwell CA, Atkinson M, Renner E (2016) Experimental approaches to studying cumulative culture. Curr Dir Psychol Sci 25(3):191195. https://doi.org/10.1177/0963721416641049

Caldwell CA, Atkinson M, Blakey KH, Dunstone J, Kean D, Mackintosh G et al (2020) Experimental assessment of capacities for cumulative culture: review and evaluation of methods. WIREs Cogn Sci 11:e1516

Colombelli-Négrel D, Hauber ME, Kleindorfer S (2014) Prenatal learning in an Australian songbird: habituation and individual discrimination in superb fairy-wren embryos. Proc R Soc B 281:20141154

Darwin CR (1872) The expression of the emotions in man and animals. John Murray, London

Davis EP, Gwynn LM, Schetter CD, Hobel C, Chicz-Demet A, Sandman CA (2007) Prenatal exposure to maternal depression and cortisol influences infant temperament. J Am Acad Child Psychiatry 46(6):737-746

Davis SJ, Vale GL, Schapiro SJ, Lambeth SP, Whiten A (2016) Foundations of cumulative culture in apes: improved foraging efficiency through relinquishing and combining witnessed behaviours in chimpanzees (Pan troglodytes). Sci Rep 6:35953

de Beaune SA (2015) L'homme et l'outil. CNRS Éditions, Paris

de Beaune SA (2016) Qu'est-ce que la préhistoire? Gallimard, Paris

Dean LG, Vale GL, Laland KN, Flynn EG, Kendal RL (2014) Human cumulative culture: a comparative perspective. Biol Rev 89(2):284-301

Fox Keller E (2010) The mirage of a space between nature and nurture. Duke University Press, Durham

Freudenthal G (1986) Atom and individual in the age of Newton: on the genesis of the mechanistic world view. Reidel, Dordrecht

Fuentes A (2016) The extended synthesis, ethnography, and the human niche: toward an integrated anthropology. Curr Anthropol 57(S13):S13-S26

Galef BG (1976) Social transmission of acquired behavior: a discussion of tradition and social learning in vertebrates. Adv Stud Behav 6:77-100

Gould SJ (1976) Human babies as embryos. Nat Hist 85(2):22-26

Gruber T, Luncz L, Mörchen J, Schuppli C, Kendal RL, Hockings K (2019) Cultural change in animals: a flexible behavioural adaptation to human disturbance. Palgrave Commun 5:64. https://doi. org/10.1057/s41599-019-0271-4

Haidle MN (2019) The origin of cumulative culture-not a single-trait event, but multifactorial processes. In: Overmann KA, Coolidge FL (eds) Squeezing minds from stones. Oxford University Press, New York, pp 128-148

Haidle MN, Bräuer J (2011) From brainwave to tradition-how to detect innovations in tool behavior. PaleoAnthropology 2011:144-153

Haidle MN, Bolus M, Collard M, Conard NJ, Garofoli D, Lombard M et al (2015) The nature of culture: an eight-grade model for the evolution and expansion of cultural capacities in hominins and other animals. J Anthropol Sci 93:43-70
Hepper P (2015) Behavior during the prenatal period: adaptive for development and survival. Child Dev Perspect 9(1):38-43

Hublin JJ (2003) Évolution des hominidés et origine du langage. In: Changeux JP (ed) Gènes et culture. Odile Jacob, Paris, pp 17-33

Hunt GR, Gray RD (2003) Diversification and cumulative evolution in New Caledonian crow tool manufacture. Proc R Soc B 270:867-874

Jesmer BR, Merkle JA, Goheen JR, Aikens EO, Beck JL, Courtemanch $\mathrm{AB}$ et al (2018) Is ungulate migration culturally transmitted? Evidence of social learning from translocated animals. Science 361:1023-1025

Kempe M, Lycett SJ, Mesoudi A (2014) From cultural traditions to cumulative culture: parameterizing the differences between human and nonhuman culture. J Theor Biol 359:29-36

Kendal JR (2011) Cultural niche construction and human learning environments: investigating sociocultural perspectives. Biol Theory 6:241-250

Kendal J, Tehrani JJ, Odling-Smee J (2011) Human niche construction in interdisciplinary focus. Philos Trans R Soc B 366:785-792

Krützen M, Willems EP, van Schaik CP (2011) Culture and geographic variation in orangutan behavior. Curr Biol 21:1808-1812

Kummer H (1971) Primate societies. Group techniques of ecological adaption. Aldine-Atherton, Chicago

Laland KN, O'Brien MJ (2011) Cultural niche construction: an introduction. Biol Theory 6(3):191-202

Laland KN, Odling-Smee J, Feldman MW (2000) Niche construction, biological evolution, and cultural change. Behav Brain Sci 23(1):131-146

Lehrman DS (1953) A critique of Konrad Lorenz' theory of instinctive behavior. Q Rev Biol 28(4):337-363

Leroi-Gourhan A (1945) Milieu et techniques. Albin Michel, Paris

Lévi-Strauss C ([1949] 1969) The elementary structures of kinship. (Bell JH, Sturmer JR, Needham R (eds) trans.). Beacon Press, Boston

Levins R, Lewontin RC (1985) The dialectical biologist. Harvard University Press, Cambridge

Lewis HM, Laland KN (2012) Transmission fidelity is the key to the build-up of cumulative culture. Philos Trans R Soc B 367:2171-2180

Löffler D (2019) Generative Realitäten. Generative Realitäten I: Die Technologische Zivilisation als neue Achsenzeit und Zivilisationsstufe Eine Anthropologie des 21 Jahrhunderts. Velbrück, Weilerswist-Metternich

Logan CJ, Breen AJ, Taylor AH, Gray RD, Hoppitt WJE (2016) How New Caledonian crows solve novel foraging problems and what it means for cumulative culture. Learn Behav 44:18-28

Lombard M (2016) Mountaineering or ratcheting? Stone Age hunting weapons as proxy for the evolution of human technological, behavioral and cognitive flexibility. In: Haidle MN, Conard NJ, Bolus M (eds) The nature of culture. Based on an interdisciplinary symposium 'The nature of culture', Tübingen, Germany. Springer, Dordrecht, pp 135-146

Lombard M, Haidle MN, Högberg A (2019) Cognition: From capuchin rock pounding to Lomekwian flake production. Camb Archaeol J 29(2):201-231

Luncz LV, Wittig RM, Boesch C (2015) Primate archaeology reveals cultural transmission in wild chimpanzees (Pan troglodytes verus). Philos Trans R Soc B 370:20140348

Luria A, Vygotsky LS (1992) Ape, primitive man and child. Essays in the history of behavior. Harvester Wheathsheaf, New York

Malafouris L (2013) How things shape the mind. MIT Press, Cambridge

McGrew WC, Tutin CEG (1978) Evidence for a social custom in wild chimpanzees? Man 13(2):234-251 
Mesoudi A, Thornton A (2018) What is cumulative cultural evolution? Proc R Soc B 285:20180712

Moon C (2017) Prenatal experience with the maternal voice. In: Filippa M, Kuhn P, Westrup B (eds) Early vocal contact and preterm infant brain development. Springer, Cham, pp 25-37

Odling-Smee FJ, Laland KN (2011) Ecological inheritance and cultural inheritance: what are they and how do they differ? Biol Theory 6:220-230

Odling-Smee FJ, Laland KN, Feldman MW (1996) Niche construction. Am Nat 147(4):641-648

Portmann A (1941) Die Tragzeit der Primaten und die Dauer der Schwangerschaft beim Menschen: Ein Problem der vergleichenden Biologie. Rev Suisse Zool 48(3):511-518

Pradhan GR, Tennie C, van Schaik CP (2012) Social organization and the evolution of cumulative technology in apes and hominins. $\mathrm{J}$ Hum Evol 63(1):180-190

Proffitt T, Luncz LV, Falótico T, Ottoni EB, de la Torre I, Haslam M (2016) Wild monkeys flake stone tools. Nature 539:85-88

Reindl E, Beck SR, Apperly IA, Tennie C (2016) Young children spontaneously invent wild great apes' tool-use behaviours. Proc R Soc B 283:20152402

Rendell L, Fogarty L, Laland KN (2011) Runaway cultural niche construction. Philos Trans R Soc B 366:823-835

Robbins MM, Ando C, Fawcett KA, Grueter CC, Hedwig D, Iwata Y et al (2016) Behavioral variation in gorillas: evidence of potential cultural traits. PLoS ONE 11(9):e0160483

Rogers EM (1995) Diffusion of innovations. The Free Press, New York

Rosenberg K, Trevathan W (1995) Bipedialism and human birth: the obstetrical dilemma revisited. Evol Anthropol 4(5):161-168

Sahlins M (2008) The Western illusion of human nature. Prickly Paradigm Press, Chicago

Sasaki T, Biro D (2017) Cumulative culture can emerge from collective intelligence in animal groups. Nat Commun 8(1):1-6

Schofield D, McGrew W, Takahashi A (2018) Cumulative culture in nonhumans: overlooked findings from Japanese monkeys? Primates 59:113-122

Schuppli C, van Schaik CP (2019) Animal cultures: how we've only seen the tip of the iceberg. Evol Hum Sci 1:e2

Schuppli C, Meulman EJM, Forss SIF, Aprilinayati F, van Noordwijk MA, van Schaik CP (2016) Observational social learning and socially induced practice of routine skills in immature wild orangutans. Anim Behav 119:87-98

Smotherman WP, Robinson SR (1990) The prenatal origins of behavioral organization. Psychol Sci 1(2):97-106

St. Clair JJH, Klump BC, van der Wal JEM, Sugasawa S, Rutz C (2016) Strong between-site variation in New Caledonian crows' use of hook-tool-making materials. Biol J Linn Soc 118(2):226-232

Stout D, Rogers MJ, Jaeggi AV, Semaw S (2019) Archaeology and the origins of human cumulative culture: a case study from the earliest Oldowan at Gona. Ethiopia Curr Anthropol 60(3):309-340

Streri A, de Hevia MD, Izard V, Coubart A (2013) What do we know about neonatal cognition? Behav Sci 3:154-169
Tennie C, Call J, Tomasello M (2009) Ratcheting up the ratchet: on the evolution of cumulative culture. Philos Trans R Soc B 364:2405-2415

Tennie C, Braun DR, Premo LS, McPherron SP (2016) The island test for cumulative culture in the Paleolithic. In: Haidle MN, Conard NJ, Bolus M (eds) The nature of culture. Based on an interdisciplinary symposium 'The nature of culture', Tübingen, Germany. Springer, Dordrecht, pp 121-133

Tennie C, Premo LS, Braun DR, McPherron SP (2017) Early stone tools and cultural transmission. Curr Anthropol 58(5):652-654

Tomasello M (1999a) The cultural origins of human cognition. Harvard University Press, Cambridge

Tomasello M (1999b) The human adaption for culture. Annu Rev Anthropol 28:509-529

Tomasello M, Kruger AC, Ratner HH (1993) Cumulative culture. Behav Brain Sci 16:495-552

Vale GL, Davis SJ, Lambeth SP, Schapiro SJ, Whiten A (2017) Acquisition of a socially learned tool use sequence in chimpanzees: Implications for cumulative culture. Evol Hum Behav 38(5):635-644

van Schaik CP, Ancrenaz M, Borgen G, Galdikas B, Knott CD, Singleton I et al (2003) Orangutan cultures and the evolution of material culture. Science 299:102-105

van Schaik CP, Ancrenaz M, Reniastoeti D, Knott CD, Morrogh-Bernard H, Nuzuar OK et al (2009) Orangutan cultures revisited. In: Wich SA, Mitra Setia T, Utami Atmoko SA, Van Schaik CP (eds) Orangutans compared: geographic variation in behavioral ecology and conservation. Oxford University Press, Oxford, pp 299-309

Wacquant L (2016) A concise genealogy and anatomy of habitus. Sociol Rev 64:64-72

West MJ, King AP (1987) Settling nature and nurture into an ontogenetic niche. Dev Psychobiol 20(5):549-562

Whitehead H, Rendell L (2014) The cultural lives of whales and dolphins. University of Chicago Press, Chicago

Whiten A (2019) Cultural evolution in animals. Annu Rev Ecol Evol Syst 50:1-22. https://doi.org/10.1146/annurev-ecolsys-11021 8-025040

Whiten A, van de Waal E (2018) The pervasive role of social learning in primate lifetime development. Behav Ecol Sociobiol 72(5):80

Whiten A, Goodall J, McGrew WC, Nishida T, Reynolds V, Sugiyama $Y$ et al (1999) Cultures in chimpanzees. Nature 399:682-685

Whiten A, Ayala FJ, Feldman MW, Laland KN (2017) The extension of biology through culture. Proc Nat Acad Sci USA 114:7775-7781

Publisher's Note Springer Nature remains neutral with regard to jurisdictional claims in published maps and institutional affiliations. 